\title{
Antibacterial Efficacy of Plasma Jet, Dielectric Barrier Discharge, Chlorhexidine, and Silver Diamine Fluoride Varnishes in Caries Lesions
}

\author{
Moritz Hertel, ${ }^{\mathrm{a}, \star \star}$ Julia Schwill-Engelhardt, ${ }^{\mathrm{b}, \star *}$ Torsten Gerling, ${ }^{\mathrm{c}}$ Klaus-Dieter \\ Weltmann, ${ }^{\mathrm{c}}$ Sandra Maria Imiolczyk, ${ }^{\mathrm{b}}$ Stefan Hartwig, ${ }^{\mathrm{d}}$ \& Saskia Preissner ${ }^{\mathrm{b}, *}$ \\ aDepartment of Oral Medicine, Dental Radiology and Oral Surgery, Charité-Universitätsmedizin \\ Berlin, Berlin, Germany; 'Department of Operative and Preventive Dentistry, Charité-Univers- \\ itätsmedizin Berlin, Berlin, Germany; 'Leibniz Institute of Plasma Science and Technology (INP \\ Greifswald), Greifswald, Germany; 'Department of Oral and Maxillofacial and Facial Plastic Surgery, \\ Johannes Wesling Hospital Minden, University Hospital of Ruhr University, Bochum, Germany

\footnotetext{
*Address all correspondence to: Dr. Saskia Preissner, Department of Operative and Preventive Dentistry, CharitéUniversitätsmedizin Berlin, Aßmannshauser Str. 4-6, 14197 Berlin, Germany; Tel.: +4930450562675, E-mail: saskia. preissner@charite.de

**These authors contributed equally to this study.
}

\begin{abstract}
The aim of this study was to assess the bactericidal efficacy of cold atmospheric plasmas (CAPs), chlorhexidine (CHX), and silver diamine fluoride (SDF) in root caries lesions (RCLs). Artificial RCLs were created in 50 human dentin samples using a computercontrolled continuous-culture biofilm model. Subsequently, CAPs (created using a plasma jet, CAP I, or a dielectric barrier discharge source, CAP II,CHX, and SDF varnishes were used in antibacterial treatment, and the number of viable bacteria was determined. To compare the different plasma sources, filament quantification was performed using an oscilloscope. All applied agents led to significantly lower counts of colony-forming units (CFUs) compared to the control ( $p \leq 0.01$; Mann-Whitney U test). The obtained logarithmic reduction factors of CAP I, CAP II, CHX, and SDF were 1.18, 0.61, 0.81, and 1.95, respectively. Only the results for SDF and CAP II differed significantly $(p=0.004)$. Filamentation imaging revealed approximately $3 \times 10^{7}$ filaments for CAP I and $3 \times 10^{5}$ for CAP II within $60 \mathrm{~s}$. Compared to the control, all investigated agents significantly reduced CFU numbers in artificial RCLs. Among those, the effect of SDF was superior to that of CAP II. All investigated agents provided antibacterial effects and may therefore be used for the noninvasive treatment of RCLs.
\end{abstract}

KEYWORDS: root caries lesion, cold atmospheric plasma, dielectric barrier discharge, tissuetolerable plasma, silver diamine fluoride, chlorhexidine, continuous-culture biofilm model

\section{INTRODUCTION}

While the caries experience is continually decreasing among children and younger adults in developed countries, ${ }^{1}$ the prevalence of root caries is increasing because of the aging population. ${ }^{2}$ Clinicians face a variety of challenges: the detection of carious lesions on root surfaces is difficult because they are often situated far cervically from present restoration margins and covered by plaque or gingiva. ${ }^{3}$ Once detected, the restorative 
treatment of these lesions is challenging because the adhesive bond is not moisture tolerant ${ }^{4}$ and alternative restoration materials, such as glass ionomer cements, often fail because of insufficient macroretention. ${ }^{5}$ As a result of marginal leakage, secondary caries is likely to appear. ${ }^{6}$ The concept of treating initial root caries lesions (RCLs) includes the application of varnishes, such as chlorhexidine (CHX) or silver diamine fluoride (SDF), ${ }^{7}$ to arrest progression. Recently, it was reported that cold atmospheric plasma (CAP) might be promising in RCL treatment. ${ }^{8}$

Cold atmospheric plasma is primarily used in dermatology to treat chronically infected wounds, ${ }^{9}$ but it is also used to stimulate healing mechanisms after surgery. ${ }^{10}$ Even though different methods to generate cold atmospheric plasma for medical applications exist, all result in the creation of a unique mixture of potential antimicrobial agents, such as reactive oxygen and nitrogen species, as well as ultraviolet radiation. ${ }^{11}$ The composition depends on the type of plasma generation and on the tuning of the operational parameters. Two core technologies have so far been emphasized: plasma jet technology, which generates plasma in the form of a jet in an inert gas; and dielectric barrier discharge (DBD) systems, which ignite plasma on top of a dielectric barrier or between a dielectric barrier and the targeted surface. ${ }^{12}$ The potential use of CAP in dentistry has been investigated in in vitro, ex vivo, and in vivo studies for the treatment of periimplantitis, ${ }^{13-16}$ infected dentin including curved root canals,,${ }^{17,18}$ infected bone,,${ }^{19,20}$ oral candidiasis, ${ }^{21}$ and enhancement of adhesive bond strength..$^{22}$

\section{A. Aims}

Focusing on conservative therapy for RCLs, the intent of the present study was to evaluate the bactericidal efficacy of CAPs (test groups) and CHX and SDF varnishes (positive control groups) in artificial RCLs. Furthermore, the aim was to compare the bactericidity of a plasma jet device with that of a dielectric barrier discharge source. The primary hypothesis was that all applied agents would result in significantly lower counts of CFUs in Lactobacillus rhamnosus compared to the negative control (brushing with no agent). The secondary hypothesis was that CAP irradiation would result in a significantly greater reduction in CFU numbers compared to CHX and SDF, regardless of the applied plasma source.

\section{MATERIALS AND METHODS}

\section{A. Sample Preparation}

Selected for sample preparation were 50 caries-free human upper canines that had been stored in $0.5 \%$ chloramine-t (Carl Roth, Karlsruhe, Germany) after extraction. The crowns and $3.0 \mathrm{~mm}$ of the apices were cut off using a 0.2-mm bandsaw (EXAKT 300 CL, EXAKT, Advanced Technologies, Norderstedt, Germany). Subsequently, one block-shaped dentin specimen from each obtained root fragment was prepared and trimmed to dimensions of $6.0 \times 4.0 \times 2.0 \mathrm{~mm}$ using a grinding machine (Labo Pol 25, 
Struers, Willich, Germany) and SiC P1000 sandpaper (Buehler, Düsseldorf, Germany). In total, 50 blocks were created and embedded in acrylic resin (Technovit 4071, Heraeus Kulzer, Wehrheim, Germany). Of the upper surface, $6.0 \times 4.0 \mathrm{~mm}$ remained exposed. The resin blocks were then polished using SiC P2500 and P4000 sandpapers (Buehler, Düsseldorf, Germany). The same resin was used subsequently to create a standardized basin-shaped repository of 20-mL capacity above the exposed dentin.

\section{B. Bacteria Cultivation}

L. rhamnosus (DSM strain 20021, Leibniz Institute DSMZ-German Collection of Microorganisms and Cell Cultures, Braunschweig, Germany) was cultivated in de Man, Rogosa and Sharpe (MRS) broth (Carl Roth, Karlsruhe, Germany) containing $10.0 \mathrm{~g} / \mathrm{L}$ peptone, $4.0 \mathrm{~g} / \mathrm{L}$ yeast extract, $8.0 \mathrm{~g} / \mathrm{L}$ beef extract, $20.0 \mathrm{~g} / \mathrm{L}$ glucose, $2.0 \mathrm{~g} / \mathrm{L}$ dipotassium phosphate, $5.0 \mathrm{~g} / \mathrm{L}$ sodium acetate, $2.0 \mathrm{~g} / \mathrm{L}$ ammonium citrate, $0.2 \mathrm{~g} / \mathrm{L}$ magnesium sulfate, $0.05 \mathrm{~g} / \mathrm{L}$ manganese sulfate, and $1.0 \mathrm{~g} / \mathrm{L}$ polysorbate 80 with a $\mathrm{pH}$ of 6.2 , and stored in an incubator (Type 6420, Thermo Electron, Langselbold, Germany) for $24 \mathrm{~h}$ at $37^{\circ} \mathrm{C}$ under aerobic conditions.

\section{Artificial Root Caries Lesions}

For demineralization, the dentin specimens were transferred to a buffered acidic solution containing 2.205-g CaCl$\times 2 \mathrm{H}_{2} \mathrm{O}$, 2.041-g $\mathrm{KH}_{2} \mathrm{PO}_{4}, 10.0-\mathrm{mL} 100 \%$ methylene diphosphonic acid, and $14.3-\mathrm{mL} 100 \% \mathrm{CH}_{3} \mathrm{COOH}$ added to $4.5 \mathrm{~L}$ of distilled water and supplemented with $10 \mathrm{M} \mathrm{KOH}$ (Merck, Darmstadt, Germany). When a pH of 5.0 was reached, the solution was stored for $48 \mathrm{~h}$ in an incubator at $37^{\circ} \mathrm{C}$ shaken at $50 \mathrm{rpm}$ (ES20 Orbital Shaker-Incubator, Biosan, Riga, Latvia). Following this, the samples were autoclaved for $15 \mathrm{~min}$ at $121^{\circ} \mathrm{C}$ and $2.1 \mathrm{bar}$ (3870 ELV, Tuttnauer, Breda, Netherlands). Subsequently, biofilms were created in a computer-controlled continuous-culture biofilm mode $1^{23-25}$ within five days at $37^{\circ} \mathrm{C}$ and $100 \%$ humidity to simulate the environmental conditions of the oral cavity. Initially, each sample was statically incubated with $20.0 \mathrm{~mL}$ of 24-h-old L. rhamnosus broth for $15 \mathrm{~min}$. Within the following $15 \mathrm{~min}, 20.0$ $\mathrm{mL}$ of fresh bacteria broth was continuously pumped (Ismatec MCP Standard, ColeParmer, Wertheim, Germany) over the dentin samples. This procedure was repeated four times, with a static interval of $225 \mathrm{~min}$ between cycles. After the fifth pump cycle, static incubation was maintained for $9 \mathrm{~h}$. Excessive broth was constantly removed from the mouth model using a peristaltic pump (Type PR1, Seko, Rieti, Italy). Every 24 h, the procedure was repeated, beginning with $15 \mathrm{~min}$ of exposure to fresh 24-hour-old bacterial cultures.

\section{Antibacterial Treatment}

After five days, the dentin specimens were harvested from the mouth model, the MRS broth was completely removed, and each sample was rinsed with $1.0 \mathrm{~mL}$ of $0.9 \%$ sterile 
$\mathrm{NaCl}$ (B. Braun Melsungen, Melsungen, Germany). The artificial RCLs were randomly divided into five groups, each containing ten specimens, and treated accordingly.

\section{Negative Control (C)}

Negative control samples were treated using a blank microbrush to remove nonadherent biofilm (Appli-Tip, Medirel, Agno, Switzerland).

\section{Test I (CAP I)}

Cold atmospheric plasma irradiation was applied for $60 \mathrm{~s}$ by constantly moving the plasma jet (kINPen MED, Neoplas Tools GmbH, Greifswald, Germany), fed with 4.3-sL/ $\mathrm{min}^{-1}$ argon, in a perpendicular position and within a distance of $8.0 \mathrm{~mm}$ to the specimen surface. An engine-driven movable laboratory bench was used to ensure homogeneous irradiation and a constant feed of $10.0 \mathrm{~mm} / \mathrm{s}^{-1}$, as recommended by the manufacturer.

\section{Test II (CAP II)}

Plasma emitted by the DBD source (PlasmaDerm, Cinogy, Duderstadt, Germany) was applied for $60 \mathrm{~s}$, whereby the electrode was kept in close contact with the sample surface. Again, an engine-driven movable laboratory bench was used to constantly move the plasma source at $5.0 \mathrm{~mm} / \mathrm{s}^{-1}$ above the RCL.

\section{Positive Control I (CHX)}

For 5 min, $0.1 \mathrm{~g}$ of a $40 \%$ CHX varnish (EC40, Biodent, Nijmegen, Netherlands) per RCL was evenly spread using a microbrush.

\section{Positive Control II (SDF)}

Both components of the $38 \%$ SDF/potassium iodide (KI) (Riva Star, SDI Dental, Dublin, Ireland) were subsequently applied to the RCLs according to the manufacturer's instructions. Application times were $5 \mathrm{~s}$ for both solutions (silver-labeled and greenlabeled).

\section{E. CFU Counts}

Following treatment, each sample was rewetted with $5.0 \mu \mathrm{L}$ of $\mathrm{NaCl}$ using a microbrush, and dentin chips were harvested from the lesions in a standardized manner using a scalpel (No. 15, Aesculap, Tuttlingen, Germany) mounted on a movable laboratory bench at a $45^{\circ}$ contact angle and at $0.7-\mathrm{N}$ contact pressure. The sampling procedure consisted of five cycles of linear removal of dentin from the center of the specimens. The obtained dentin chips were subsequently transferred into sterile 2.0-mL jars (Eppendorf, Ham- 
burg, Germany), each containing $1.0 \mathrm{~mL}$ of $\mathrm{NaCl}$, and vortexed for $10 \mathrm{~s}$ (Vortex Genie 2, Bender \& Hobein, Zürich, Switzerland). After serial dilution and vortexing for $3 \mathrm{~s}, 100.0$ $\mu \mathrm{L}$ of each dilution was dispersed onto MRS agar plates (Oxoid, Wesel, Germany). After $48 \mathrm{~h}$ of cultivation at $37^{\circ} \mathrm{C}$ under aerobic conditions, the CFUs were enumerated.

\section{F. Statistical Analysis}

Statistical analysis was performed using the Mann-Whitney U test (IBM SPSS 24.0, IBM, Armonk, IL). The significance threshold was defined as $p=0.05$.

\section{G. Scanning Electron Microscopy}

After treatment, the surfaces of the artificial RCLs were comparatively examined using a scanning electron microscope (SEM) (CamScan Maxim 2040S, CamScan Electron Optics, Cambridgeshire, U.K.). At a magnification of up to $2000 \times$, secondary and backscatter electron images (SEI/BEI) were recorded applying acceleration voltages of $10-20 \mathrm{kV}$.

\section{H. Comparative Filament Quantification}

In order to compare the CAP I and CAP II plasma devices, fast and sensitive electrical discharge analysis was performed to quantify the number of discharges within a time frame. A fast oscilloscope (Waverunner 640Zi, Teledyne LeCroy, Chestnut Ridge, NY) at $4.0-\mathrm{GHz}$ resolution was used in combination with a current probe (CT-1, Tektronix, Beaverton, $\mathrm{OR}$ ) at $1.0-\mathrm{GHz}$ resolution connected to a conducting surface representing the sample at the respective distance ( $8.0 \mathrm{~mm}$ for CAP I and $1.0 \mathrm{~mm}$ for CAP II) and connected to the HV line for CAP I (CAP II had no measurement access). In addition, for CAP I a high-voltage probe (1:1000 Tektronix P6015 A, Tektronix, Beaverton, OR) was used to measure the HV signal, whereas for CAP II a 1:100 voltage probe (P008, Teledyne LeCroy, Chestnut Ridge, NY) was used as an antenna to assess the relative voltage slope.

\section{RESULTS}

\section{A. CFU Counts}

After applying CAP I, CAP II, CHX, and SDF, the median $\log \mathrm{CFU} / \mathrm{mL}$ were $6.18,6.74$, 6.54 , and 5.40, respectively. The obtained CFU counts of all groups were significantly lower compared to the control samples $(7.35 \log \mathrm{CFU} / \mathrm{mL}$; $p$ CAP I vs. $\mathrm{C}<0.0001$, CAP II vs. $\mathrm{C}=0.007$, $\mathrm{CHX}$ vs. $\mathrm{C}=0.002$, $\mathrm{SDF}$ vs. $\mathrm{C}<0.0001$ ). The resulting logarithmic reduction factors for CAP I, CAP II, CHX, and SDF were calculated (Fig. 1).

Comparison of the investigated antibacterial agents revealed that CFU counts were significantly more reduced by SDF compared to CAP II $(p=0.004)$. No significant differences were found between all other groups $(p>0.05)$. 


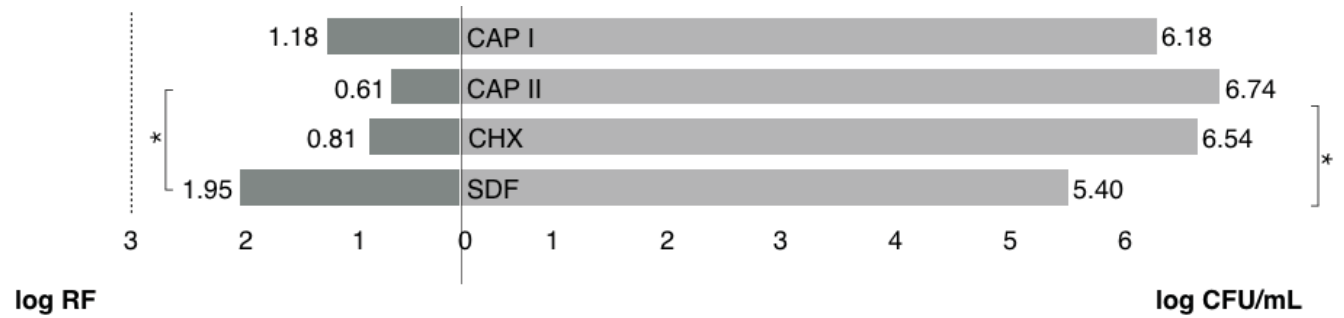

FIG. 1: Logarithmic reduction factors and $\log \mathrm{CFU} / \mathrm{ml}$ of CAP I, CAP II, CHX, and SDF $\left({ }^{*} p \leq\right.$ $0.05)$

\section{B. Scanning Electron Microscopy}

Scanning electron microscopic (SEM) imaging revealed that all specimens were covered with dense biofilms, including rod-shaped bacteria, regardless of the applied treatment (Fig. 2). Different from the control and both CAP groups, the surfaces were additionally covered with an amorphous coating after applying CHX and with granules of two different sizes after using SDF.

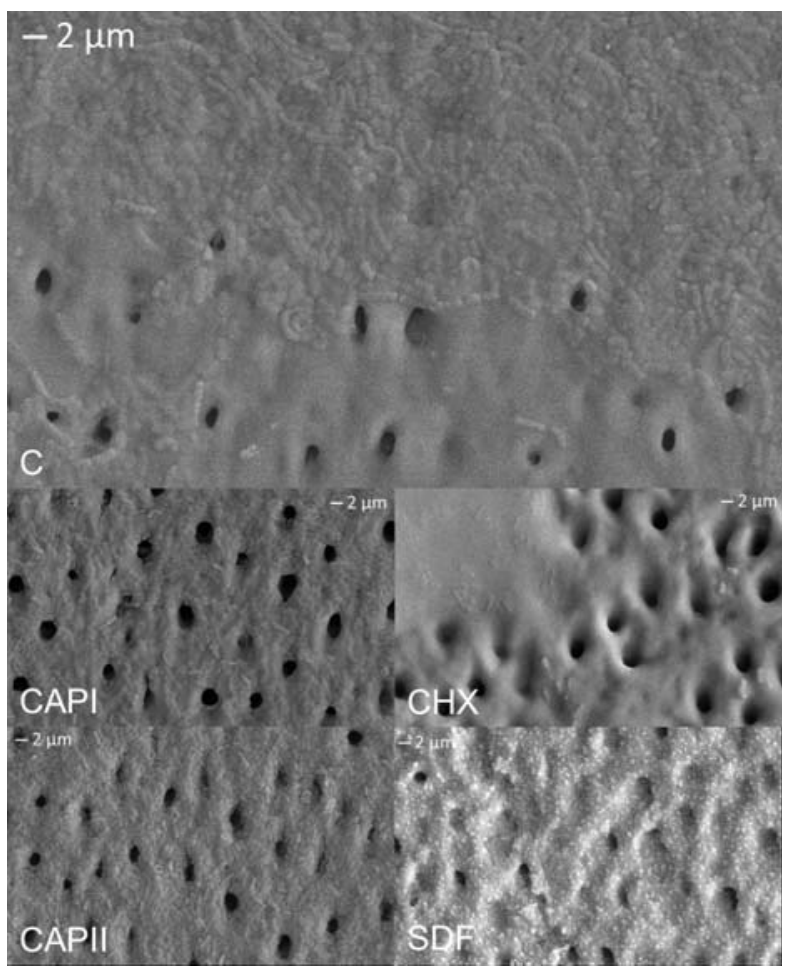

FIG. 2: Backscatter scanning electron images $(2,000 \times) . \mathrm{C}=$ control group sample; CAP I = sample after treatment with CAP I; CAP II = sample after treatment with CAP II; CHX = sample after treatment with $\mathrm{CHX}$; SDF = sample after treatment with SDF 


\section{Filamentation Imaging}

CAP I showed a periodically applied high voltage of $2-3 \mathrm{kV}$ peak to peak together with the measured displacement current of the high-voltage electrode (Fig. 3). In each period, a discharge current peak was observed, indicated by a black arrow in the enlargement (Fig. 3). It was previously shown that each period is indeed a single filament/streamer leaving the orifice of the device and entering the surrounding air. ${ }^{23}$ With an operation frequency of approximately $1 \mathrm{MHz}$ and a $50 \%$ duty cycle, a total of $5 \times 10^{5}$ filaments per second or 3 $\times 10^{7}$ filaments per $60 \mathrm{~s}$ of treatment were generated with CAP I. The characteristic electrical signals of CAP II discharge are different, given that each microdischarge (MD) can be identified by fast current oscillations (black arrows in Fig. 3). For a source similar to CAP II, there have as yet been no single-shot investigations of the filaments for one ignition phase; rather, only time-averaged pictures have been published. ${ }^{26}$ Because DBD discharges are known to have a statistical distribution of discharge peaks, ${ }^{27} 1,000$ discharge cycles were analyzed with respect to discharge currents. The resulting average number of MDs was approximately 15 per cycle for CAP II, which corresponds to 5,000 MDs/s. For the treatment time of $60 \mathrm{~s}$, the total number of MDs was approximately $3 \times 10^{5}$.

\section{IV.DISCUSSION}

The aim of the present study was to assess the antibacterial efficacy of two cold atmospheric plasma systems, CHX, and SDF in artificial RCLs. The primary hypothesis was confirmed in that the application of all investigated agents led to significantly lower CFU numbers compared to the negative control. However, the detected logarithmic reduction factors were limited to $>1$ to $<2$ (CAP I and SDF) and $>0$ to $<1$ (CAP II and $\mathrm{CHX}$ ); also, disinfection quality, which requires logarithmic reduction factors $>5$, was not found in any group. In this regard, findings from other studies indicate that CAP
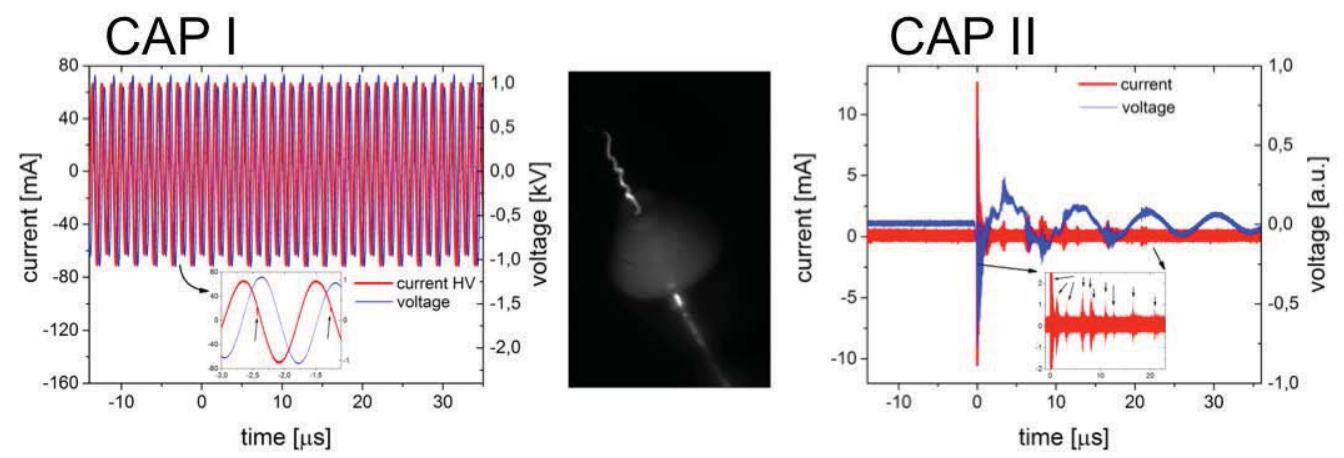

FIG. 3: Electrical measurement of discharge current and voltage of CAP I and CAP II. CAP I: current measured on supply line; CAP II: current measured on treated surface. Middle: single shot acquisition of a filament exiting a jet device similar to CAP I

Volume 8, Issue 1, 2018 
might be more efficient in combination with additional antimicrobial agents and/or the mechanical removal of biofilms. ${ }^{8,16,28}$ Despite cold plasma being a promising approach to conservatively treating RCLs, its adjuvant use may be considered in addition to mechanical debridement. Moreover, the kinetics of the antimicrobial effects of CAP remain unknown. It may be assumed that the long-term effects might be inferior or even absent compared to varnishes, which provide both slow drug release and resistance regarding oral clearance. This issue may be taken up in future research.

The secondary hypothesis was not confirmed given that the obtained CFU numbers after CAP II treatment were significantly higher, at least when compared to the numbers after SDF treatment. Although the plasma jet generated up to $3 \times 10^{7}$ filaments in the direction of the specimen surface, the DBD source created up to $3 \times 10^{5}$ MDs in connection with the specimen surface-in the case of CAP II acting as the second active electrode. It must be pointed out that the applied plasma jet uses inert argon as the operating gas, which is mixed with the surrounding ambient air to generate a broad spectrum of reactive components. In contrast, the investigated DBD device operates directly in ambient air and therefore provides a more nitrogen-based gas chemistry triggered by the MDs. ${ }^{29}$ As a result, a direct comparison of the number of filaments and MDs can be seen as a first insight into the various antimicrobial potentials of the different systems. However, these facts must be discussed with respect to the triggered chemistry in the respective gas phase. So far, the results show that the type and the operating parameters of the applied plasma source may influence its therapeutic potential. Future studies might focus on the differences in plasma ignition techniques and compare their respective impact on relevant parameters, especially in vivo.

Compared to the negative control, CAP I, CAP II, CHX, and SDF significantly reduced CFU numbers in artificial RCLs. Among these agents, the effect size of SDF was superior to that of CAP II. Within the limitations of the present study, it can be concluded that all applied antibacterial agents provide antibacterial effects and can therefore be used in conservative treatment of RCLs.

\section{COMPLIANCE WITH ETHICAL STANDARDS}

Ethical approval: This article does not contain any clinical studies including humans or animals performed by any of the authors. Formal approval was granted by the institutional ethical review committee of the Charité-Universitätsmedizin Berlin (EA4/102/14).

Informed consent: The patients from whom the utilized teeth were obtained gave written informed consent.

\section{ACKNOWLEDGMENTS}

The authors would like to thank Mrs. Carola Schweynoch for her help in the microbiology lab and Dr. Herbert Renz for taking SEM micrographs. The CAP devices were on loan from Neoplas Tools GmbH (Greifswald, Germany) and PlasmaDerm (Cinogy, Duderstadt, Germany). 


\section{REFERENCES}

1. Schiffner U, Hoffmann T, Kerschbaum T, Micheelis W. Oral health in German children, adolescents, adults and senior citizens in 2005. Commun Dent Health. 2009;26(1):18-22.

2. Eke PI, Wei L, Borgnakke WS, Thornton-Evans G, Zhang X, Lu H, McGuire LC, Genco RJ. Periodontitis prevalence in adults $>/=65$ years of age, in the USA. Periodontol 2000. 2016;72(1):76-95.

3. Hayes M, Da Mata C, Cole M, McKenna G, Burke F, Allen PF. Risk indicators associated with root caries in independently living older adults. J Dent. 2016;51:8-14.

4. Chibinski AC, Stanislawczuk R, Roderjan DA, Loguercio AD, Wambier DS, Grande RH, Reis A. Clinical versus laboratory adhesive performance to wet and dry demineralized primary dentin. Am J Dent. 2011;24(4):221-5.

5. Burke FJ. Dental Materials: what goes where? Class V restorations. Dental Update. 2015;42(9):82930, 33-6, 39.

6. Ludlow S, Farmer S, Donaldson M, Tantbirojn D, Versluis A. Microleakage of resin-modified glass ionomer restorations with selective enamel etching. Oper Dent. 2014;39(4):E154-9.

7. Deutsch A. An alternate technique of care using silver fluoride followed by stannous fluoride in the management of root caries in aged care. Spec Care Dentist. 2016;36(2):85-92.

8. Pierdzioch P, Hartwig S, Herbst SR, Raguse JD, Dommisch H, Abu-Sirhan S, Wirtz HC, Hertel M, Paris S, Preissner S. Cold plasma: a novel approach to treat infected dentin-a combined ex vivo and in vitro study. Clin Oral Investig. 2016;20(9):2429-35.

9. Ulrich C, Kluschke F, Patzelt A, Vandersee S, Czaika VA, Richter H, Bob A, Hutten JV, Painsi C, Hüge R, Kramer A, Assadian O, Lademann J, Lange-Asschenfeldt B. Clinical use of cold atmospheric pressure argon plasma in chronic leg ulcers: a pilot study. J Wound Care. 2015;24(5):196, 8-200, 202-203.

10. Hartwig S, Doll C, Voss JO, Hertel M, Preissner S, Raguse JD. Treatment of wound healing disorders of radial forearm free flap donor sites using cold atmospheric plasma: a proof of concept. J Oral Maxillofac Surg. 2017 Feb;75(2):429-35.

11. Haertel B, Strassenburg S, Oehmigen K, Wende K, von Woedtke T, Lindequist U. Differential influence of components resulting from atmospheric-pressure plasma on integrin expression of human HaCaT keratinocytes. Biomed Res Int. 2013;2013:761451.

12. von Woedtke T, Reuter S, Masur K, Weltmann KD. Plasmas for medicine. Physics Reports. 2013; 530(4):291-320.

13. Preissner S, Wirtz HC, Tietz AK, Abu-Sirhan S, Herbst SR, Hartwig S, Pierdzioch P, Schmidt-Westhausen AM, Dommisch H, Hertel M. Bactericidal efficacy of tissue tolerable plasma on microrough titanium dental implants: an in-vitro-study. J Biophotonics. 2016;9(6):637-44.

14. Duske K, Jablonowski L, Koban I, Matthes R, Holtfreter B, Sckell A, Nebe JB, von Woedtke T, Weltmann KD, Kocher T. Cold atmospheric plasma in combination with mechanical treatment improves osteoblast growth on biofilm covered titanium discs. Biomat. 2015;52:327-34.

15. Idlibi AN, Al-Marrawi F, Hannig M, Lehmann A, Rueppell A, Schindler A, Jentsch H, Rupf S. Destruction of oral biofilms formed in situ on machined titanium (Ti) surfaces by cold atmospheric plasma. Biofouling. 2013;29(4):369-79.

16. Rupf S, Idlibi AN, Marrawi FA, Hannig M, Schubert A, von Mueller L, Spitzer W, Holtmann H, Lehmann A, Rueppell A, Schindler A. Removing biofilms from microstructured titanium ex vivo: a novel approach using atmospheric plasma technology. PLoS One. 2011;6(10):e25893.

17. Li Y, Sun K, Ye G, Liang Y, Pan H, Wang G, Zhao Y, Pan J, Zhang J, Fang J. Evaluation of cold plasma treatment and safety in disinfecting 3 -week root canal Enterococcus faecalis biofilm in vitro. J Endod. 2015;41(8):1325-30.

18. Ureyen Kaya B, Kececi AD, Guldas HE, Cetin ES, Ozturk T, Oksuz L, Bozduman F. Efficacy of endodontic applications of ozone and low-temperature atmospheric pressure plasma on root canals infected with Enterococcus faecalis. Lett Appl Microbiol. 2014;58(1):8-15.

Volume 8, Issue 1, 2018 
19. Abu-Sirhan S, Hertel M, Preissner S, Wirtz HC, Herbst SR, Pierdzioch P, Raguse JD, Hartwig S. Bactericidal efficacy of cold plasma in processed bone. A new approach for adjuvant therapy of medication-related osteonecrosis of the jaw? Clin Plasma Med. 2016;4(1):9-13.

20. Eisenhauer P, Chernets N, Song Y, Dobrynin D, Pleshko N, Steinbeck MJ, Freeman TA. Chemical modification of extracellular matrix by cold atmospheric plasma-generated reactive species affects chondrogenesis and bone formation. J Tissue Eng Regen Med. 2016;10(9):772-82.

21. Preissner S, Kastner I, Schutte E, Hartwig S, Schmidt-Westhausen AM, Paris S, Preissner R, Hertel M. Adjuvant antifungal therapy using tissue tolerable plasma on oral mucosa and removable dentures in oral candidiasis patients: a randomised double-blinded split-mouth pilot study. Mycoses. 2016;59(7):467-75.

22. Kim JH, Han GJ, Kim CK, Oh KH, Chung SN, Chun BH, Cho BH. Promotion of adhesive penetration and resin bond strength to dentin using non-thermal atmospheric pressure plasma. Eur J Oral Sci. 2016;124(1):89-95.

23. Iseni S, Reuter S, Schmidt-Bleker A, Weltmann K-D. Flow and discharge development in an argon atmospheric pressure plasma jet observed by ICCD and PLIF imaging. IEEE Trans on Plasma Sci. 2014;42(10):2458-9.

24. Schwendicke F, Dorfer C, Kneist S, Meyer-Lueckel H, Paris S. Cariogenic effects of probiotic Lactobacillus rhamnosus GG in a dental biofilm model. Caries Res. 2014;48(3):186-92.

25. Sissons CH, Cutress TW, Hoffman MP, Wakefield JS. A multi-station dental plaque microcosm (artificial mouth) for the study of plaque growth, metabolism, $\mathrm{pH}$, and mineralization. J Dent Res. 1991;70(11):1409-16.

26. Ayan H, Staack D, Fridman G, Gutsol A, Mukhin Y, Starikovskii A, Fridman A, Friedman G. Application of nanosecond-pulsed dielectric barrier discharge for biomedical treatment of topographically non-uniform surfaces. J Physics D: Appl Physics. 2009;42(12):125202.

27. Wagner HE, Brandenburg R, Kozlov KV, Sonnenfeld A, Michel P, Behnke JF. The barrier discharge: basic properties and applications to surface treatment. Vacuum. 2003;71(3):417-36.

28. Herbst SR, Hertel M, Ballout H, Pierdzioch P, Weltmann KD, Wirtz HC, Abu-Sirhan S, Kostka E, Paris $\mathrm{S}$, Preissner S. Bactericidal efficacy of cold plasma at different depths of infected root canals in vitro. Open Dent J. 2015;9:486-91.

29. Lu X, Naidis GV, Laroussi M, Reuter S, Graves DB, Ostrikov K. Reactive species in non-equilibrium atmospheric-pressure plasmas: generation, transport, and biological effects. Physics Rep. 2016;630: $1-84$. 